\title{
Espacios rituales y cultura material en un sitio arqueológico Humahuaca-Inca (Quebrada de Humahuaca, Jujuy, Argentina)
}

\author{
María Beatriz CREMONTE \\ CONICET - Facultad de Humanidades y Ciencias Sociales, Universidad de Jujuy (Argentina) \\ beacre@imagine.com.ar \\ María Soledad GHEGGI \\ CONICET - Facultad de Filosofía y Letras, Universidad de Buenos Aires (Argentina) \\ solelingheggi@yahoo.com.ar
}

Recibido: 10 de diciembre de 2010

Aceptado: 21 de febrero 2011

\section{RESUMEN}

Esquina de Huajra es un asentamiento arqueológico ubicado en el sector centro- sur de la Quebrada de Humahuaca (extremo septentrional del noroeste de Argentina) cuya cultura material permite caracterizarlo como Humahuaca-Inca. En este trabajo analizamos los materiales, la espacialidad y características de los entierros hallados, mayoritariamente secundarios, que conforman verdaderos osarios en recintos cuadrangulares, dentro de estructuras circulares o bien como entierros directos en vasijas ordinarias. Los ajuares son también variados, llamando especialmente la atención la presencia de cráneos de aves y de polvos de colores (rojo, amarillo, verde, naranja y azul). La perduración de materiales incaicos para la época en la que la presencia española debería ser efectiva en la zona indicaría la continuación de un modo de vida establecido bajo la administración incaica, sin alteraciones notorias, así como la vigencia de las redes de interacción preexistentes.

Palabras clave: Quebrada de Humahuaca, enterramientos, culto a los antepasados.

\section{Ritual Space and Material Culture in a Humahuaca-Inca Archaeological Site (Quebrada de Humahuaca, Jujuy, Argentina)}

\begin{abstract}
Esquina de Huajra is an archaeological site from the South-Central sector of Quebrada de Humahuaca (northern area of Northwest Argentina) which can be characterized from its material culture as a Humahuaca-Inca settlement. In this work we analyze materials, spatiality and characteristics of burials. Most interments are secondary, some of them composing ossuaries in rectangular shaped units, in circular structures or as primary interments in ordinary vessels. Mortuary offerings are also varied, calling special attention the presence of bird crania and pigments (red, yellow, green, orange and blue). The permanence of Inca material culture in moments when Spanish presence should be effective in the area would indicate the continuation of a lifestyle established under Inca administration that was not notably altered, as well as the efficacy of preexisting interaction networks.
\end{abstract}

Key words: Quebrada de Humahuaca, burials, ancestor cult.

Sumario: 1. Introducción. 2. Esquina de Huajra en contexto. 3. Descripción de los entierros analizados. 4. Rituales mortuorios: manipulación del cuerpo y espacio funerario. 5. Reflexiones finales: interpretando la evidencia. 6. Referencias bibliográficas. 


\section{Introducción}

La generación de un área de entierro es el resultado visible de una serie de conductas humanas relacionadas con la muerte que podemos englobar bajo el término de «prácticas mortuorias», entendidas como los procedimientos realizados por los allegados de un difunto para alcanzar el objetivo final del ritual mortuorio, es decir que el difunto se incorpore al mundo de los muertos y que sus deudos se reintegren a la vida social. Éstas pueden tener un correlato material (manipulación del cadáver, ofrendas, construcción o elección del lugar de depósito del cuerpo, etc.) o no material (gestos, peregrinaciones, oraciones, cantidad de personas presentes, etc.) (Bartel 1982).

Desde el punto de vista arqueológico, contamos con un registro que corresponde a los correlatos materiales del ritual mortuorio, como son los restos óseos, el acompañamiento mortuorio, las ofrendas realizadas y la construcción y/o emplazamiento de la tumba. Si consideramos que las prácticas sociales sólo adquieren relevancia cuando se sitúan en un eje espacio/ tiempo (Giddens 1984; Mizoguchi 1993: 223-235) y que particularmente para las prácticas mortuorias, los individuos enterrados pueden ser entendidos como «transportadores» de un significado ritual y de una memoria social (Turner 1995 [1969]), podemos interpretarlas como un medio de reproducción de la estructura social que informa acerca de aspectos religiosos, económicos y políticos de una comunidad (Carr 1995: 186).

Si bien los rituales mortuorios en los Andes son variados y complejos, comparten en muchos casos la característica de que la muerte es vista tanto como una experiencia personal como comunitaria donde el mundo de los muertos no está separado del mundo de los vivos, ya que los muertos «viven» en relación permanente con sus familiares y con la comunidad; a los difuntos se los puede llamar siempre que sea necesario, porque sus almas (ajayus) son protectoras, consejeras y mensajeras (Aláez García 2001: 173-178; Bascopé Caero 2001: 271-277). Este modelo de concepción de los muertos les otorga un rol importante en tanto en cuanto ellos tienen voluntad e influyen sobre los vivos (Bloch y Parry 1982; Buikstra y Charles 1999; Parker Pearson 1993).

Desde este punto de partida -a través de diferentes líneas de evidencia provenientes de los entierros de Esquina de Huajra- intentamos aproximarnos a aspectos rituales de las prácticas mortuorias llevadas a cabo en un contexto de importantes cambios sociales, como pudo haber sido el de la anexión de este territorio al Imperio Inca y el momento de contacto inicial con los conquistadores españoles. Se comentarán cuatro entierros que hemos denominado Tumbas 1, 2, 3 y 4, considerando a cada una de ellas como el lugar donde se depositaron los cadáveres y sus acompañamientos mortuorios, pudiendo aportar información sobre las construcciones simbólicas hechas alrededor del cuerpo y en el espacio en el que interactúa el mismo (Barrett 2001: 141-164). 


\section{Esquina de Huajra en contexto}

Esquina de Huajra (1.990 msnm) es un asentamiento localizado en el sector centrosur de la quebrada de Humahuaca, estratégicamente ubicado frente a la entrada de la quebrada de Huajra, uno de los accesos naturales más directos e importantes hacia los valles orientales (Figura 1). Las dataciones radiométricas y la cultura material asociada indican que habría sido ocupado entre la Fase Inca (1430-1536 d.C.) y la Humahuaca Colonial o Hispano Indígena (1536 hasta aproximadamente 1630 d.C., fecha en que comienzan a efectivizarse las encomiendas españolas en la zona) (Cuadro 1). Se trata de una instalación de dimensiones mucho más pequeñas que las del poblado vecino «Pucará de Volcán» pero con una mayor «riqueza» y variedad de los elementos que integran sus registros arqueológicos, reflejados en la vajilla cerámica, en los objetos de metal, en sus entierros y acompañamientos funerarios.

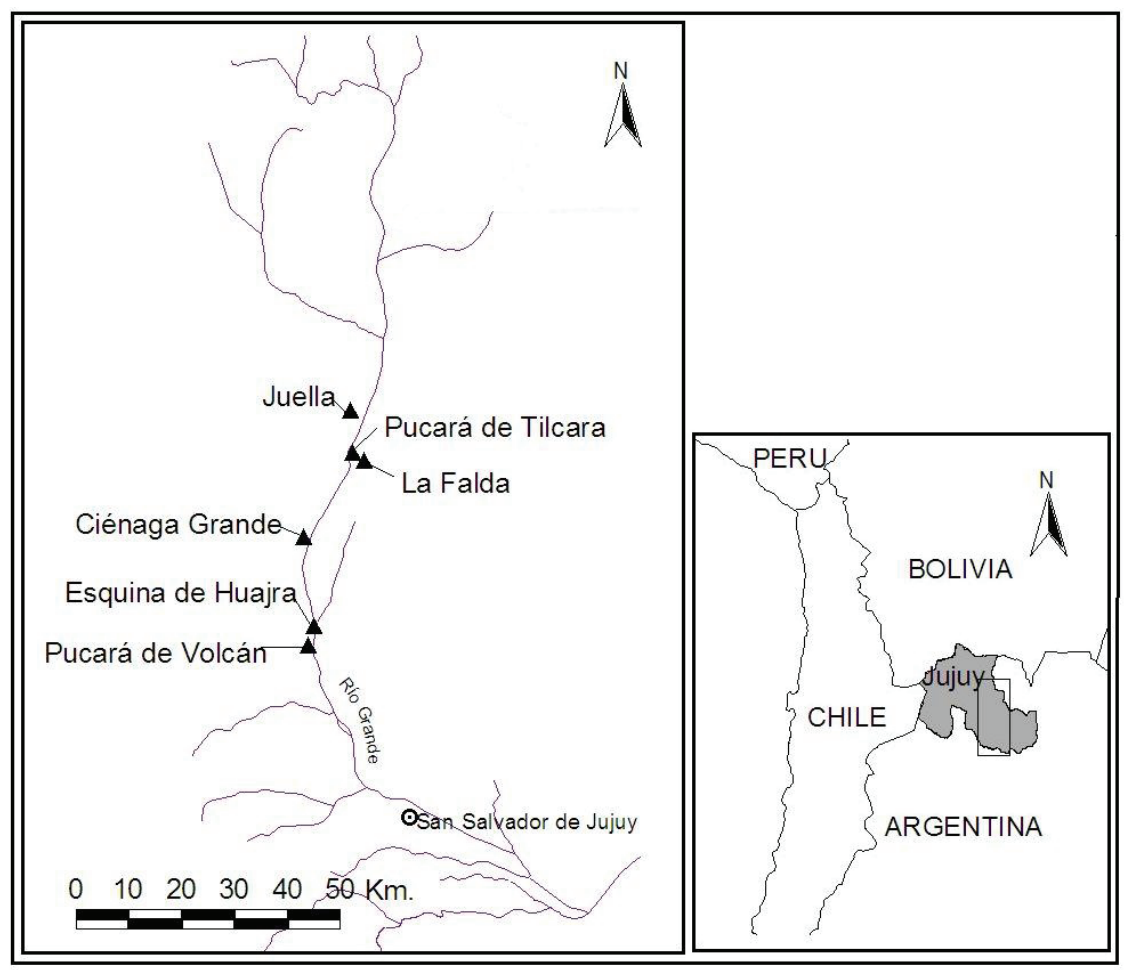

Figura 1: Mapa de la región con los sitios mencionados en el texto.

Las excavaciones ${ }^{1}$ se realizaron en tres niveles (Terraza I, Terraza II y Terraza III, desde la inferior a la superior) sobre la ladera Norte del cerro donde se concentran

1 En el año 2001 se llevaron a cabo tareas de rescate arqueológico en este sitio debido al impacto provocado por las obras de ingeniería en este tramo de la Ruta Internacional $\mathrm{N}^{\circ} 9$. 
Cuadro 1: Dataciones radiocarbónicas obtenidas para Esquina de Huajra

\begin{tabular}{|c|c|c|c|}
\hline $\begin{array}{l}\text { Número } \\
\text { laboratorio }\end{array}$ & Material utilizado / Proveniencia & $\begin{array}{l}\text { Edad radiocar- } \\
\text { bónica (A.P.) }\end{array}$ & $\begin{array}{c}\text { Edad calibrada } \\
\text { (d.C.) } \pm 2 \sigma \text { (oxcal) }\end{array}$ \\
\hline Beta 193319 & Carbón / Terraza I & $340 \pm 55$ & $1455-1796$ \\
\hline Beta 206919 & Carbón / Tumba 3 (Terraza III) & $280 \pm 50$ & $1496-1952$ \\
\hline Arizona AA88375 & Carbón / Terraza III-Piso (C 21) & $393 \pm 82$ & $1400-1664$ \\
\hline UGA 16200 & Óseo humano / Tumba 1 (Terraza III) & $550 \pm 40$ & $1318-1463$ \\
\hline Geochron 32577 & Óseo humano / Tumba 2 (Terraza III) & $450 \pm 60$ & $1419-1627$ \\
\hline Geochron 32576 & Óseo humano / Tumba 3 (Terraza III) & $320 \pm 50$ & 1460-1799 \\
\hline
\end{tabular}

las construcciones (muy poco visibles en superficie). El área excavada en la Terraza I correspondería a un sector del patio de una unidad doméstica, hallándose un fogón, fragmentos de vasijas cerámicas, una piedra de moler con su mano y material lítico y faunístico. En el conjunto cerámico están claramente representadas las formas incaicas más típicas de las provincias del imperio: ollas de cocina con pie, platos y cántaros vinculados al consumo de chicha (aríbalos). En la Terraza II (media) solo se registraron algunos muros de contención; este sector habría funcionado como un espacio de circulación interna. En la Terraza III (la más alta) se ubicaron áreas residenciales y de enterramiento. Los cuatro entierros excavados muestran variaciones en las técnicas constructivas, en las modalidades de inhumación y en sus acompañamientos mortuorios. Con excepción de la Tumba 1, todas las demás se concentraban -muy próximas entre sí- en el extremo norte de la Terraza III (Figura 2). Describimos a continuación los contextos funerarios hallados.

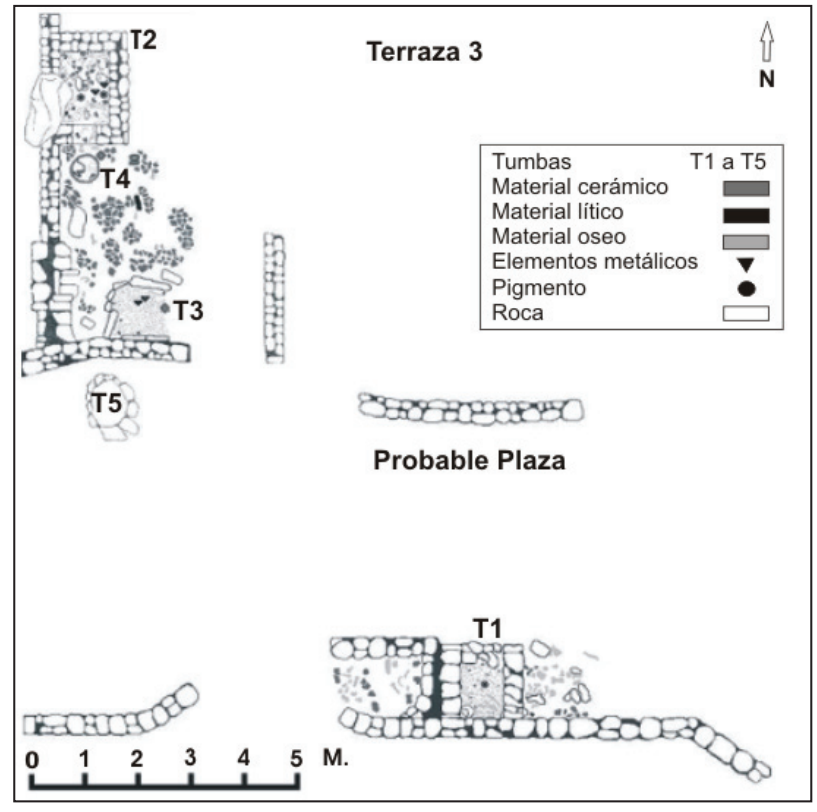

Figura 2: Ubicación de los entierros y materiales en el sector funerario hallado en Esquina de Huajra. 


\section{Descripción de los entierros analizados}

\subsection{Tumba 1}

Consiste en un sepulcro sobreelevado de planta rectangular $(1,10 \mathrm{~m} \times 0,55 \mathrm{~m})$. Las paredes son dobles de piedra y sus bloques fueron unidos con barro batido y pequeños guijarros (Figura 3). La pared oeste era parte de un muro de contención, con refuerzo en banqueta.

Este entierro contenía los restos de cuatro individuos adultos y un perinato depositados en forma secundaria. El análisis osteológico indicó que los individuos adultos participaron durante su vida en importantes actividades físicas, tal como se evidencia a partir de la marcada presencia de enfermedad articular degenerativa en la columna, miembros superiores e inferiores. Asimismo, presentan diversas patologías orales (caries, pérdida de piezas dentales antemortem y periodontitis) y no se registraron patologías nutricionales (Gheggi 2005).

El ajuar fúnebre estaba compuesto por un vasito cerámico y fragmentos de cerámica de tipo ordinario correspondientes a una o dos vasijas, un pequeño terrón de pigmento rojo (hematina: $\mathrm{Fe}_{2} \mathrm{O}_{3}$ ), una placa aplanada y alisada de esquisto, una punta de hueso confeccionada a partir de un metapodio de camélido y un tubo también de hueso (probablemente utilizado para inhalar alucinógenos). Esta tumba arrojó el fechado más antiguo (550 \pm 50 A.P.); el material datado corresponde al húmero derecho de una mujer adulta, indicando la fecha aproximada de su muerte, pero no la del entierro secundario que pudo ser posterior. Basamos esto último en las características constructivas de la tumba y en la presencia de una punta pulida confeccionada en hueso de camélido (un elemento recurrente en los registros arqueológicos del momento Hispano-Indígena) (Figura 4).

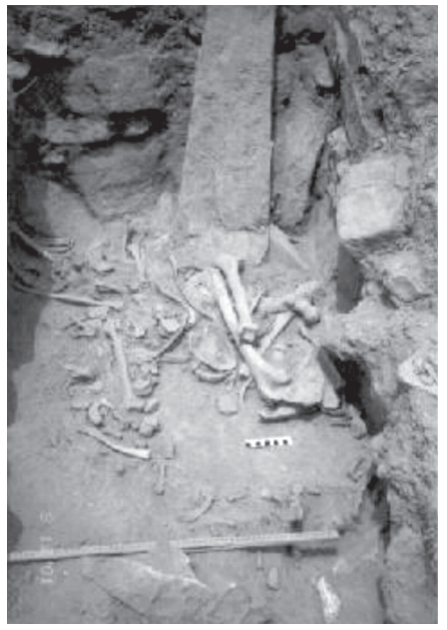

Figura 3: Nivel de los hallazgos en la Tumba 1. Se observa que los restos óseos están desarticulados y depositados en un mismo evento.

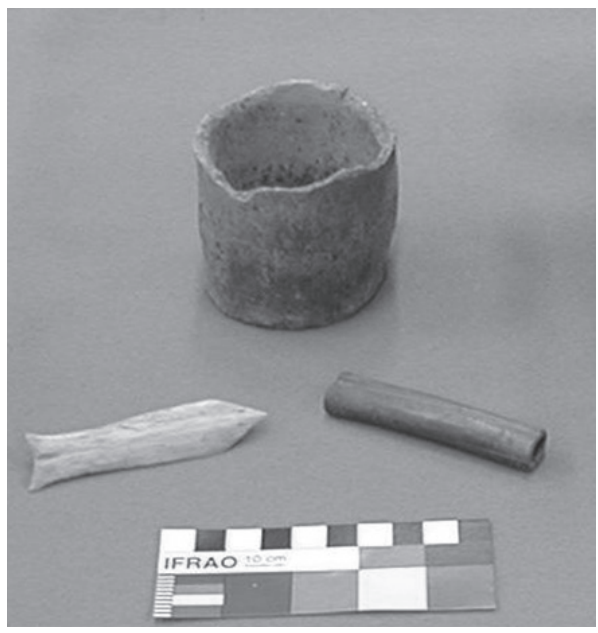

Figura 4: Algunos de los ítems del acompañamiento mortuorio de la Tumba. 


\subsection{Tumba 2}

Se trata de un sepulcro sobre-elevado de planta rectangular $(2,50 \mathrm{~m} \times 1,50 \mathrm{~m})$ con una entrada de $0,40 \mathrm{~m}$ de ancho (Figura 5). En el interior de esta estructura y entre los 0,25 y $0,45 \mathrm{~m}$ de profundidad, se hallaron los restos óseos de siete individuos, de los cuales cuatro son subadultos con edades entre los 3 y 10 años, algunos de los cuales presentan patologías bucales (caries) (Gheggi 2005-2006).

Entre los restos se encontró casi un centenar de cuentas de collar, 83 de las cuales fueron hechas en hueso y otras en roca carbonatada, lutita negra y turquesa (Figura 6). También acompañaban a los restos óseos fragmentos de una pinza de depilar y de un colgante de bronce estannífero, dos panes de pigmento azul (azurita molida: $\mathrm{Cu}_{3}(\mathrm{OH})_{2}\left(\mathrm{CO}_{3}\right)_{2}$ ), polvo verde (atacamita: $\mathrm{Cu}_{2} \mathrm{Cl}(\mathrm{OH})_{3}$ ) y amarillo (oropimente: $\mathrm{As}_{2} \mathrm{~S}_{3}$ ), y dos cráneos de patos criollos (Cairina Moschata s. p.). Para las ofrendas de alimentos sólidos o semisólidos y de bebidas se usó un platito del estilo local Humahuaca Negro sobre Rojo y al menos 14 vasijas cerámicas locales y no locales representadas por numerosos fragmentos (Cremonte et al. 2006-2007). De esta tumba se fechó un fragmento de fémur derecho de un subadulto obteniéndose el resultado de $450 \pm 60$ AP.

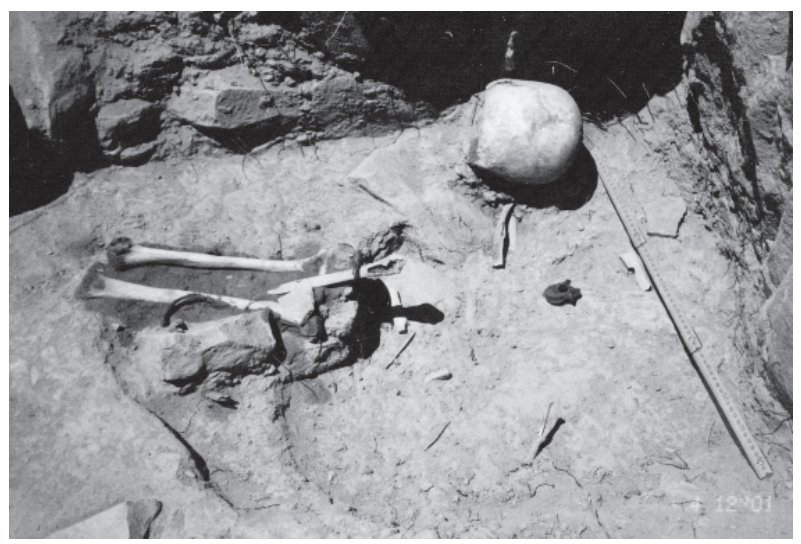

Figura 5: Nivel base de la Tumba 2.
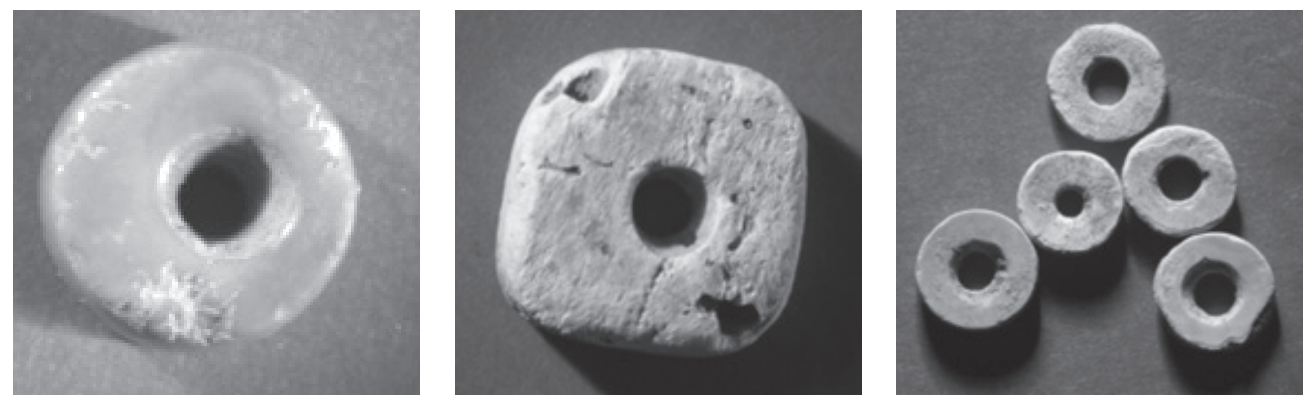

Figura 6: Cuentas de collar de diverso tipo halladas en la Tumba 2. 


\subsection{Tumba 3}

Esta unidad funeraria es de planta semicircular $(1,25 \mathrm{~m}$ x $0,70 \mathrm{~m})$ conformada por una hilera de lajas colocadas de canto y fijadas con barro batido (Figura 7). Aunque de forma y construcción diferente a las tumbas anteriores, es también un rasgo positivo de aproximadamente $0,40 \mathrm{~m}$ de altura por encima del piso de ocupación. Esta estructura alojaba el entierro secundario de una mujer de aproximadamente 40 años. El análisis osteológico permitió concluir que sufrió un proceso infeccioso específico, activo en el momento de la muerte, que involucró la porción distal de la tibia derecha, posiblemente relacionado con una lesión penetrante. Además, presentaba importante desarrollo de los músculos de la muñeca de ambos brazos, seguramente como resultado de actividades continuas de molienda, amasado y/o tejido.

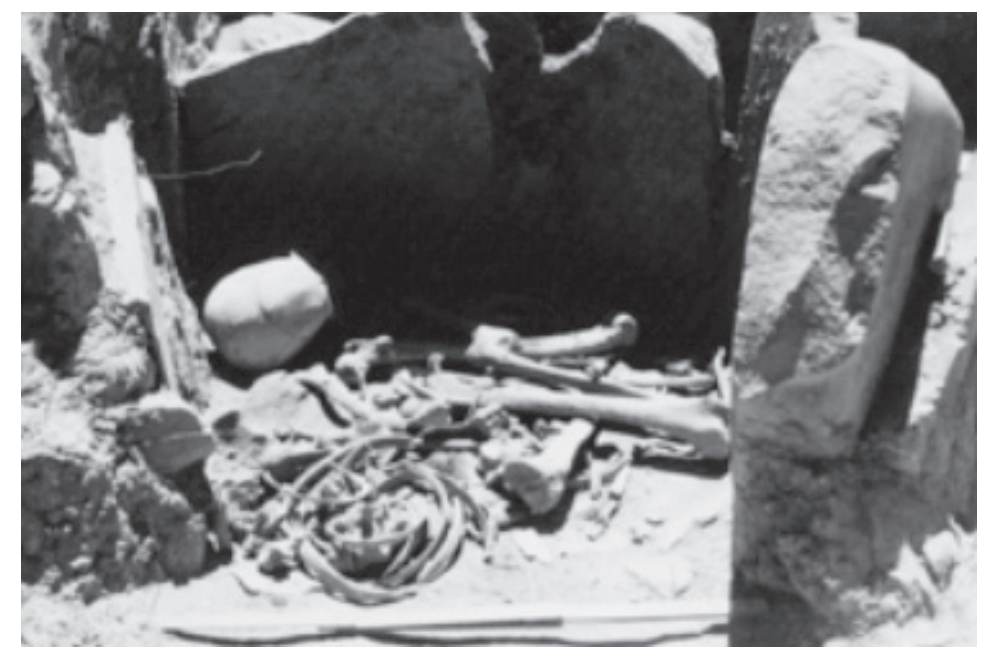

Figura 7: Estructura funeraria denominada Tumba 3. Se aprecian los restos óseos desarticulados.

El ajuar estaba integrado por un plato negro pulido (Figura 8), algunos huesos de camélido y 38 fragmentos cerámicos de diferentes estilos locales y foráneos. Por lo menos unas seis vasijas cerámicas habrían integrado el ajuar fúnebre, así como también 2 topu de metal (alfileres usados en la vestimenta femenina), uno de ellos confeccionado con una aleación de plata y cobre (Figura 8). Este último no sería de manufactura local, ya que los objetos realizados con esta aleación son muy escasos en el noroeste de Argentina (Angiorama 2003). También se halló polvo de color naranja (rejalgar: AsS) esparcido entre los huesos. Contamos con dos dataciones cronométricas para este entierro, una realizada sobre un fragmento de fémur izquierdo del único individuo hallado que arrojó un resultado de $320 \pm 50$ A.P. y la otra del carbón vegetal encontrado en el piso de la tumba, que correspondería a alguna ofrenda quemada durante el rito fúnebre y que resultó en una datación de $280 \pm 50$ A.P. 

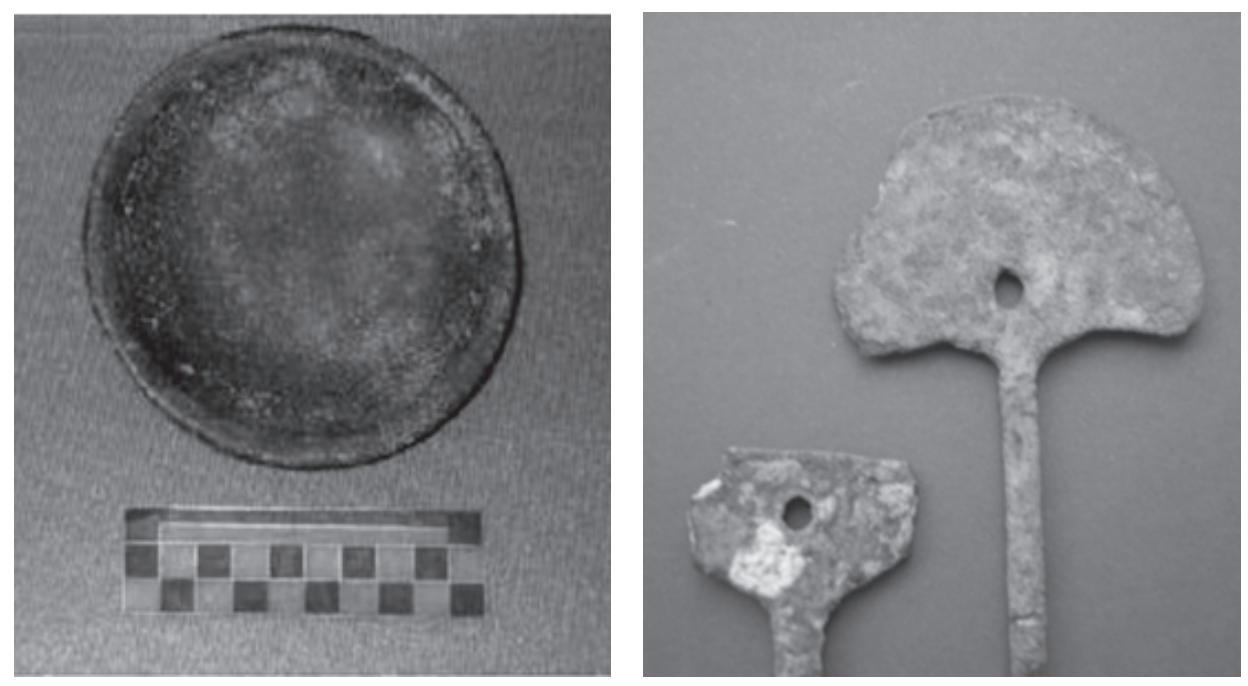

Figura 8: Plato negro pulido y topus hallados en la Tumba 3.

\subsection{Tumba 4}

Corresponde a un entierro primario depositado en el piso de un recinto doméstico (Figura 9). En el interior de una vasija grande Marrón Alisada se hallaron los restos de un niño de 7 años de edad al momento de morir y de un perinato de 38-40 semanas de gestación. El niño presentaba bandas hipoplásicas en la dentición permanente y de hipocalcificación en la dentición decidua, ambos indicadores relacionados con eventos de estrés reiterados pero no específicos.

El cuello de la vasija habría sido extraído para facilitar la ubicación de los niños y del ajuar en su interior. Apoyado sobre la boca de la vasija se encontró un cántaro pequeño (aríbalo) con sedimento y carbón en su interior, seguramente utilizado para quemar alguna ofrenda. En el interior de la vasija grande (urna) el ajuar fúnebre estaba compuesto por 2 cinceles y fragmentos de una pinza de depilar, todos de bronce estannífero; 1 cántaro de cerámica local Rosada Pulida (aríbalo) muy similar al descrito; 1 plato y una tinaja decorada con banderines verticales en negro sobre engobe rojo (motivo característico Humahuaca -Inka). Además, se hallaron fragmentos de al menos otras tres vasijas cerámicas y una escudilla decorada en Negro sobre Rojo con bandas oblicuas de líneas paralelas, idéntica a otra hallada a menos de 1 metro de distancia de este entierro y que habría formado parte del acompañamiento mortuorio estando en una posición similar a la del cántaro mencionado en primer término. Se trata de un caso de «piezas gemelas» semejante a otros registrados en entierros prehispánicos de la región y es notable el carácter doble de los elementos del ajuar (dos cinceles, dos cántaros, dos escudillas) para dos niños (Figura 10). Esta tumba corresponde a la modalidad usual de entierro de niños en vasijas y, aunque no contamos con dataciones cronométricas de los restos óseos, el fechado de carbón del piso de la Terraza III, muy próximo a la inhumación, arrojó un resultado de $393 \pm 82$ A.P. 


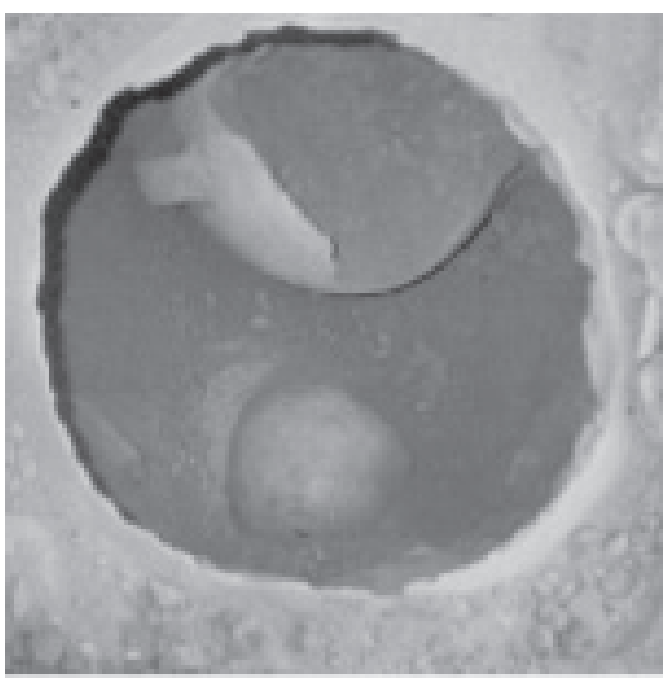

Figura 9: Entierro en urna denominado Tumba 4.

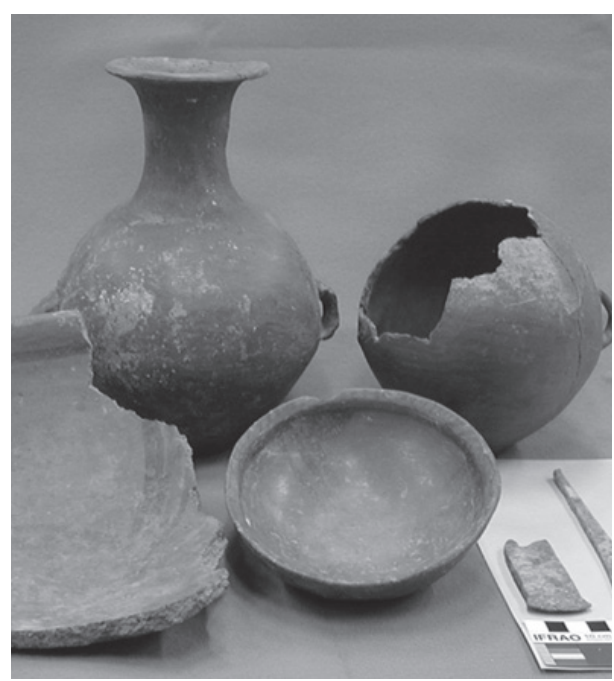

Figura 10: Ejemplares del acompañamiento funerario de la Tumba 4.

Los materiales hallados en el lugar donde se concentran las Tumbas 2, 3 y 4 consisten en un cuchillo de bronce estannífero, fragmentos de mineral de cobre, abundantes fragmentos de vasijas cerámicas similares a las encontradas en los otros sectores del sitio y material lítico que sugiere actividades destinadas a la elaboración de instrumentos (Chaparro e.p.). A juzgar por los mismos y considerando las dataciones obtenidas (Cuadro 1), este sector se trataría de un espacio doméstico donde se efectuó el entierro correspondiente a la Tumba 4, ubicada por debajo del piso de ocupación. Posteriormente habría sido remodelado con la construcción de las Tumbas 2 y 3 . La primera a su vez, muy similar a la Tumba 1 (construida a escasos 10 metros de distancia hacia el sureste).

Lamentablemente no se detectaron estructuras que permitieran identificar unidades de habitación. Tampoco se localizaron muros delimitando el espacio ocupado por los entierros, a modo de cementerio, aunque estos últimos pudieron desaparecer ya que este sector del sitio actualmente se encuentra muy distorsionado por efecto del desmoronamiento de la barranca norte del cerro. Sin embargo, resulta significativo que entre la Tumba 1 y las otras excavadas existe un espacio abierto que pudo funcionar como una kancha o plaza (ver Figura 2). Desde aquí se tiene una visión panorámica del fondo de valle hacia el norte y de la entrada a la gran quebrada de Huajra hacia el oriente. En este espacio comunitario, aunque de dimensiones restringidas (debido a la topografía del terreno), pudieron realizarse diferentes ceremonias y entre ellas los rituales mortuorios. Además, debieron ser notables las construcciones sobreelevadas donde se efectuaron los entierros secundarios, especialmente las Tumbas 1 y 2 a modo de torres de piedra cuadrangulares. 


\section{Rituales mortuorios: manipulación del cuerpo y espacio funerario}

En este momento nos preguntamos por lo que nos informan las evidencias halladas en los entierros de Esquina de Huajra en relación con las afirmaciones vertidas en la introducción y con los cambios sociales acaecidos durante el lapso de ocupación del sitio. Creemos que la manipulación de los cuerpos y la generación de un espacio mortuorio particular pueden ser elementos iluminadores en este sentido.

Con respecto al primer tema, la bibliografía existente aporta elementos para pensar que esta práctica se relaciona con el culto a los ancestros, en el sentido de que permite el acceso continuo a los restos de personas consideradas importantes en una sociedad (Gluckman 1937, citado en Morris 1991: 150).

En el Noroeste Argentino, la práctica de movimiento y tratamiento de partes óseas se registra desde el Arcaico. Un ejemplo es la colección Torres-Aparicio, con dataciones para Humahuaca del 3350-3150 d.C. En el sitio Peña de las Trampas, en Antofagasta de la Sierra (Provincia de Catamarca), se halló un pozo conteniendo los restos de al menos cuatro individuos juveniles acompañados por importante ajuar, con una cronología de cerca de 6.000 años d.C., en un contexto muy similar al hallado en Inca Cueva 4 (Provincia de Jujuy) con una cronología más antigua aún (ca. 7000-8000 d.C.). Ya en pleno Formativo, hay ejemplos de tumbas abiertas, vaciadas y vueltas a sellar en Punta de la Peña 9 (Antofagasta de la Sierra) y El Remate 1 (Amaicha del Valle, Provincia de Tucumán) (Aschero 2007: 102). Aschero proporciona la siguiente interpretación con la que estamos totalmente de acuerdo: «Una explicación posible tiene que ver con la demarcación de territorios. Si uno intenta leerlo desde la perspectiva de una territorialidad muy temprana, podría significar gente marcando espacios con reliquias de sus muertos, inaugurando prácticas que han de continuar en el tiempo, vinculándose con el papel que han de jugar luego los ancestros y su litomorfosis demarcando el paisaje social» (Aschero 2007: 102).

Específicamente, en el sector centro-sur de la quebrada de Humahuaca, contamos con datos sobre la funebria del Pucará de Volcán que muestran unos pocos casos de entierros secundarios. Uno de ellos se ubica debajo del piso de una vivienda y consiste en una urna ubicada dentro de una tumba circular, conteniendo restos óseos quemados y removidos (Suetta 1969). Otros casos se registraron en uno de los sectores con alta concentración de tumbas, pero no debajo de viviendas. Un hallazgo particular es la Tumba «B», localizada en el sector central del sitio, que contenía los restos de al menos siete individuos, los cuales se hallaban removidos y presentaban numerosos objetos como acompañamiento (Suetta: 5). En cambio, en el cercano sitio incaico Ciénaga Grande, de las 52 unidades funerarias trabajadas por Salas (1945), ninguna corresponde a un entierro secundario.

Los entierros secundarios hallados hasta el momento en Esquina de Huajra son variados y diferentes a los conocidos para los distintos sectores de la Quebrada de Humahuaca (Casanova 1942; Cigliano 1960; Mendonça et al. 1997; Nielsen y Boschi 2007) lo que plantea una serie de interrogantes que creemos nos llevan al segundo tema planteado previamente y que pueden estar relacionados con la generación de un espacio particular para el culto a los ancestros, dando cuenta del rol de los antepasados en la vida de los vivos (Parker Pearson 1993), una idea presente en el escenario 
andino tanto en tiempos del Inca, como se desprende a través de las fuentes (Arriaga 1968 [1621]; Cobo 1964), como hasta la actualidad (Onofre Mamaní 2001; van Kessel 2001).

En este sentido, la cercanía geográfica de los rasgos funerarios de Esquina de Huajra reflejaría la creación de un lugar destinado al culto a los ancestros realizado muy probablemente en el espacio abierto intermedio entre las tumbas, donde, debido a las dimensiones del mismo (50 $\mathrm{m}^{2}$ aproximadamente), se congregaría un grupo de individuos aunque no una gran audiencia. El hecho de que las tumbas sean rasgos positivos (i.e. que se encuentren sobre elevados) remite a su impacto visual desde diferentes sectores, sobre todo desde el norte. En la actualidad, el cementerio de Tumbaya (localidad donde se encuentre el sitio arqueológico Esquina de Huajra) se ubica sobre una meseta sobre elevada que es altamente visible cuando uno transita por la Ruta Nacional No 9 (Figura 11). Además, el hecho de que estos sepulcros no hayan contado con una tapa a modo de sello, abundaría en la interpretación de que debían estar siempre disponibles para la introducción y/o remoción de restos óseos y para el reemplazo o renovación de ofrendas.

Tal como plantea Nielsen (2006: 83) para los sepulcros sobre elevados de Los Amarillos, aquí también las Tumbas 1 y 2 pudieron ser «monumentos a los antepasados» a las que se acudía regularmente para «dar de comer y beber a los difuntos».

Ahora bien, ¿qué sucede si comparamos, en principio, el área de entierro de Esquina de Huajra con aquellas presentes en sitios próximos de la quebrada? En el Pucará de Volcán, además de los entierros localizados en el interior de las viviendas, se observan al menos dos áreas formales de entierro segregadas del núcleo habitacional (el agrupamiento más numeroso se encuentra hacia el sector NO del espolón

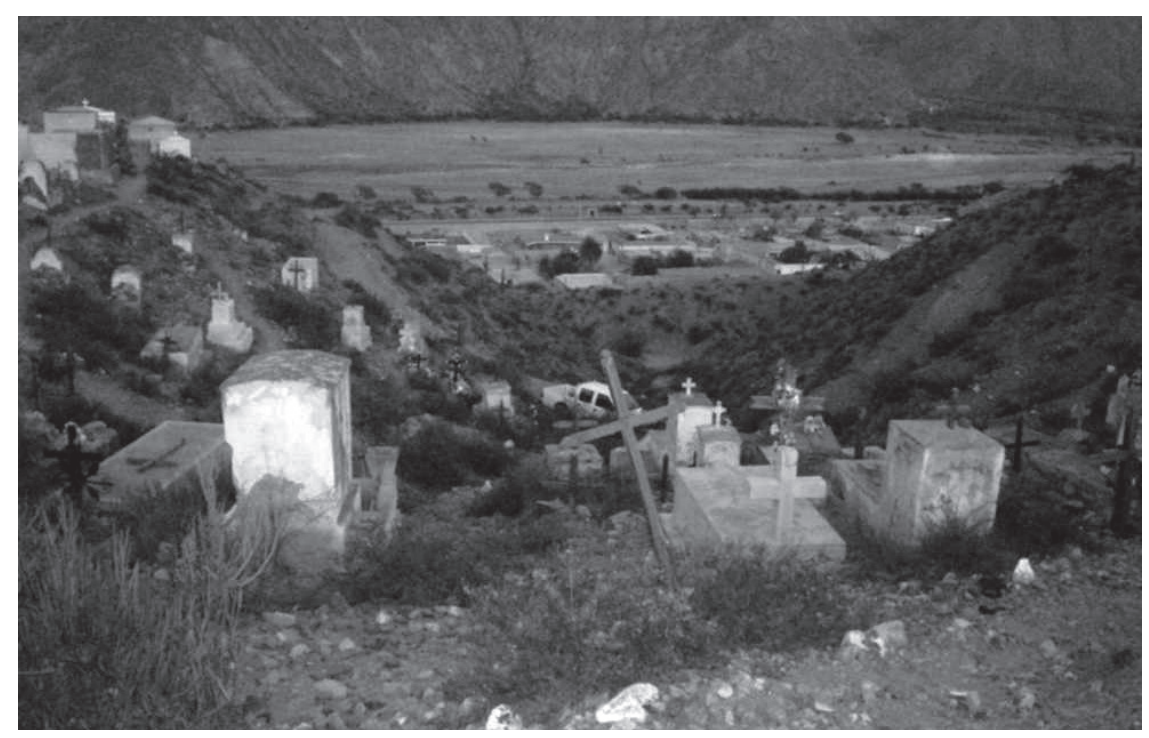

Figura 11: Cementerio actual de Tumbaya emplazado en una topografía similar a la de la Terraza 3 del sitio arqueológico Esquina de Huajra. 
residencial y cuenta con 70 tumbas, otro sector se ubica a continuación del montículo occidental y está separado del área habitacional por un muro). No sucede lo mismo en Ciénaga Grande, donde Salas (1945) registró todos los entierros en el interior de las habitaciones.

Hacia el norte, el Pucara de Tilcara presenta una situación similar a la del Pucara de Volcán (con cementerio segregado y enterramientos en viviendas), mientras que La Falda es un cementerio de la época Hispano-Indígena con estructuras funerarias del tipo «forma de bota» (Mendonça et al.1997).

Un caso particular lo constituye Los Amarillos, donde se registraron entierros secundarios en diferentes ubicaciones. Uno de los hallazgos se realizó en tres sepulcros sobreelevados construidos con muros de adobe que contenían los restos de al menos dos individuos que se encontraban quemados y removidos. En este mismo sector (denominado Complejo A) se halló un pozo conteniendo los restos de al menos cinco individuos que carecían de los huesos de los miembros inferiores y de cráneo. La interpretación de Nielsen (2007; Boschi: 110) acerca de esta plataforma, que data del Período de los Desarrollos Regionales (ca. 1200 d.C.) es que se trata de un «[...] escenario para ceremonias públicas centradas en los cuerpos de tres difuntos junto con sus sepulcros y ofrendas». Además, se han hallado restos óseos sin conexión en el Complejo B, y en varios recintos (recinto 301, 302, 320 y cista 2 del recinto 402) del Complejo E (Seldes 2006: 58 y ss.).

Sin lugar a dudas, la contextualización de los hallazgos de Esquina de Huajra con los realizados hasta el momento en el resto de la quebrada pone de manifiesto el rol primordial de los ancestros en la vida de las comunidades prehispánicas que habitaron la región, demostrando el importante papel de estos en la conformación de la experiencia cotidiana de sus habitantes. Existe la propuesta de que en muchas sociedades los muertos se colocan al servicio de la creación ideológica y de la manipulación social (Bloch y Parry 1982; Cannon 1989; Parker Pearson 1993), y que el mantenimiento de un área formal de enterramiento se relaciona con la presencia de grupos corporativos que poseen derechos de explotación sobre un determinado territorio (Goldstein 1981: 61). En este sentido, la presencia de áreas formales de enterramiento definiría el territorio de un grupo y el derecho a su usufructo.

\section{Reflexiones finales: interpretando la evidencia}

Los enterramientos hallados hasta el momento en Esquina de Huajra corresponderían más bien a un ritual comunal, poniendo de relieve la práctica de una o varias ceremonias consecutivas en relación con la manipulación de los restos de los difuntos.

En este sentido y considerando el amplio lapso temporal que abarcan los entierros de Huajra, creemos que los rituales mortuorios llevados a cabo ponen en evidencia dos cuestiones principales. Por un lado, que la gente que enterró a los muertos en este sitio preservó ciertas conductas de la esfera mortuoria comunes a momentos previos, como es el entierro conjunto de individuos adultos y subadultos y de diferente sexo. Por otro lado, la diferencia más notable es la magnitud que alcanzó en Esquina de Huajra el enterramiento secundario, señalando quizás la resignificación de este 
espacio funerario como $w^{\prime} a k a^{2}$ tal vez en estrecha relación con la demarcación de territorios ante movimientos poblacionales. Por consiguiente nos preguntamos, ¿el mantenimiento de estas prácticas rituales puede ser indicativo de una conducta de resistencia de la población ante el avance de la conquista hispana? (Garay de Fumagalli y Cremonte 2007).

A diferencia de lo que ocurre en el cementerio hispano-indígena de La Falda de Tilcara (Bordach 2006), en Esquina de Huajra no aparecen elementos europeos, aunque sus fechados parecen indicar que Huajra continuó siendo ocupado hasta el siglo XVII.

Planteamos como posibilidad que, debido a lo tardío de la penetración efectiva española en el área, no se reflejen los elementos europeos en los momentos tempranos de esa ocupación (Garay de Fumagalli y Cremonte 2007). Las cámaras mortuorias de La Falda son muy diferentes a las de Huajra y la cerámica, aunque con formas y decoraciones Humahuaca-Inca, son de manufactura más descuidada.

A pesar de las diferencias mencionadas, los acompañamientos mortuorios incluyen algunos elementos comunes, característicos de los contextos hispano-indígenas (puntas de proyectil de hueso, topus o alfileres de metal, bolsitas con pigmentos, tubos insuflatorios, esqueletos enteros o partes de diversos tipos de aves). También encontramos elementos comunes con los de cementerios de la misma época de otras regiones del noroeste de Argentina, como el de Cachi Adentro (Provincia de Salta); nos estamos refiriendo a las abundantes «pastillas» de hematita utilizadas para cubrir con polvo rojo a todos los elementos de los enterramientos y a diseños cerámicos como el de la escudilla No 364 (Tarragó 1984: 143-185) similar al de las escudillas «gemelas» asociadas a la Tumba 4 de Huajra.

Una mención especial merece el hallazgo de los panes de pigmentos y polvos de colores en las tumbas de Huajra que formaban parte del ajuar pero que no fueron utilizados para colorear los huesos (Cremonte et al. 2009). A ello se suma la presencia de grandes piedras planas con adherencias de hematita halladas en los pisos domésticos de las Terrazas I y III.

¿Qué nos indica su presencia? Estos elementos como ofrendas mortuorias pudieron estar vinculados a diferentes prácticas sociales. Por un lado, la costumbre de colorear los cadáveres con polvos rojos se remonta en los Andes Centrales al período Precerámico, y óxidos de cobre como la hematita o con contenido de arsénico y azufre como el rejalgar y el oropimente (mezclados con algo de arcilla), pudieron servir para decorar distintos tipos de objetos (por ejemplo keros de madera). Es decir que estos pigmentos podrían relacionarse con producciones artesanales llevadas a cabo por los difuntos en vida. Sin embargo, no podemos dejar de mencionar que el azul y el rojo eran los colores reales del incario. En su Descripción del Perú, Tucumán, Río de La Plata y Chile, Reginaldo de Lizárraga, al hablar de la provincia de los Chichas y Lipes (ubicadas en el sur del actual territorio de Bolivia), señala la importancia que tenían las piedras medicinales usadas en todo el reino del Perú, especialmente $\ll \ldots$ la una de color azul, con la cual se curan cualesquier llagas viejas con no poca

2 Tanto en aymara como en quechua, el vocablo w'aca hace referencia a una divinidad, deidad o cosa sagrada. 
mordacidad, con la cual las castra y en breve sanan...» (Lizárraga 2002 [1607]: 231). Esto nos hace pensar que los polvos de color rojo, amarillo y azul encontrados en la Tumba 2 pudieron haber tenido un uso medicinal sin eliminar otros significados y/o funciones. Tal como señala Siracusano, en la época colonial y como derivación de la prehispánica, los polvos de colores fueron entendidos como portadores de poder divino, y en su amplio espectro simbólico servían para sanar, para identificar jerarquías y definir desigualdades sociales, y prometían riquezas a quienes sabían manipularlos (Siracusano 2005: 269). Polvos de diferentes colores (procedentes de cerros venerados como w'acas) tenían un poder sagrado y muchos de ellos fueron luego utilizados en la pintura colonial andina. En este sentido cobra especial relevancia el azul de azurita $\left(\mathrm{Cu}_{3}(\mathrm{OH})_{2}\left(\mathrm{CO}_{3}\right)_{2}\right)$, mineral de cobre ampliamente utilizado para pintar los mantos de las vírgenes. Se sabe que, por lo menos desde fines del siglo XVII, existía una ruta de comercialización de estos polvos de colores entre la puna de Jujuy y Potosí (en actual territorio de Bolivia), y que Matheo Pisarro (pintor de la puna jujeña) empleó azurita en su «Coronación de la Virgen por la Santísisma Trinidad», en la «Virgen de la Almudena» y en el manto de Dios de «la Virgen de la Merced con santos mercedarios». A través de la ruta mencionada, seguramente preexistente desde épocas prehispánicas, podía accederse a estos pigmentos, resignificados en una nueva expresión artística pero que continuarían transmitiendo silenciosamente su poder sagrado ancestral.

Por otro lado, especialmente la atacamita $\left(\mathrm{CuCl}[\mathrm{OH}]_{3}\right)$, un mineral de cobre que procedería del área cuprífera del norte de Chile, se considera como una evidencia del tráfico de minerales y objetos metálicos en el Area Circumpuneña Meridional. Asimismo, su dimensión simbólica se manifiesta en las ofrendas de polvo de atacamita y de cuentas de minerales de cobre (malaquita, turquesa), encontradas en contextos de tránsito caravanero (rutas, abras, cerros asociados a caminos) y en el entierro (Cista 2) de Los Amarillos (Angiorama 2007; Nielsen 2004).

Es llamativa la asociación, en Esquina de Huajra, de minerales de cobre cuya procedencia podría ser local o no (ya sea molidos -como la atacamita- o en pequeños panes como la azurita), con cuentas de turquesa, objetos metálicos de cobre nativo, de bronce estannífero o aleaciones de plata y cobre, junto con alfarería de producción no local. También es notable la elevada proporción de vasijas procedentes de las tierras altas, como las del estilo Yavi/Chicha y Casabindo Pintado (con las características inclusiones blancas en sus pastas), y muchas otras con componentes volcánicos que indican diferentes procedencias; un ejemplo es el estilo Inka Pacajes del altiplano sur boliviano. Pero también la alfarería de Huajra revela contactos directos con grupos de los valles orientales más bajos. Estos elementos están dando cuenta de la amplitud de las redes de intercambio y relaciones sociales en las cuales estuvo involucrado este asentamiento (Cremonte et al. 2008). Reforzando esta misma idea, se han hallado objetos de obsidiana procedentes de tres fuentes diferentes, dos de las cuales corresponden a la Puna Salteña y la tercera permanece aún desconocida (Chaparro e.p.).

En el esquema de la política económica que aplicó la administración incaica en la zona, Esquina de Huajra habría sido un punto clave en la articulación económica entre zonas ecológicas diferentes; asimismo pudo cumplir un rol importante en el control de la mano de obra aportada por la población del Pucara de Volcán en la 
explotación y distribución de los bienes procedentes de los valles orientales (alucinógenos, maderas, plumas, etc.) y quizás también en la estructuración y sostenimiento de la frontera oriental incaica (Cremonte 2007: 331-344). Muchos de estos procesos parecen haber continuado hasta la primera mitad del siglo XVII.

Esquina de Huajra muestra la perduración de símbolos materiales incaicos en tiempos en los que la presencia española debería ser efectiva en la zona. Su cultura material indicaría la continuación de un modo de vida establecido bajo la administración incaica, sin alteraciones notorias, así como la vigencia de las redes de interacción preexistentes. Esto puede explicarse al considerar que, en la quebrada de Humahuaca, las verdaderas modificaciones en el cambio de vida indígena comienzan a partir de 1596 y se van consolidando hacia 1630, cuando se crean los primeros pueblos coloniales y funcionan efectivamente las encomiendas (Sánchez 1996; Sica 2006). En el marco del amplio espectro de situaciones de contactos más o menos directos entre las comunidades locales y los españoles, Huajra parece reflejar un reforzamiento de su identidad aborigen a través del despliegue material de atributos incaicos y de concentrar espacialmente a sus muertos reelaborando antiguas prácticas del culto a los ancestros, quizás como una estrategia de resistencia ante nuevos e incontrolables cambios.

Agradecimientos: Para este estudio se contó con la aportación de subsidios otorgados a los Proyectos ANPCYT-PICT 14425 (dirigido por Verónica Williams) y ANPCYT PICT 01538 (dirigido por M. B. Cremonte). Nuestro agradecimiento a E. Tonni y A. Cione (CONICETUNLP) por la identificación de los restos de aves. A M. Garay de Fumagalli y L. Laguna por la co-dirección de los trabajos de campo, así como a miembros del Pueblo Originario de Tumbaya y alumnos de la FHyCS (Universidad Nacional de Jujuy) que participaron en los mismos.

\section{Referencias bibliográficas}

Alaéz García, Argimiro

2001 «Duelo andino: sabiduría y elaboración de la muerte en los rituales mortuorios». Chungará 33 (2): 173-178. doi: 10.4067/S0717-73562001000200002

Angiorama, Carlos I.

2003 Producción y circulación de objetos de metal en la Quebrada de Humahuaca en momentos Prehispánicos Tardios $(900-1535$ d. c.). Tesis doctoral inédita. Tucumán: FCN e Instituto Miguel Lillo.

2007 «¿Una ofrenda 'caravanera' en Los Amarillos? Minerales y tráfico de bienes en tiempos prehispánicos», en Producción y circulación de bienes en el sur andino, A. Nielsen et al., comps., tomo 2, pp. 383-392. Córdoba: Editorial Brujas.

ArRiaga, Pablo J.

1968 The Extirpation of Idolatry in Peru [1621]. Lexington: University of Kentucky Press. 
Aschero, Carlos A.

2007 «Comentario a la Mesa 'Interacciones Surandinas. Aspectos económicos, políticos e ideológicos'», en Sociedades Precolombinas Surandinas. Temporalidad, interacción y dinámica en el ámbito de los Andes Centro-Sur, V. Williams et al., eds., pp. 99-108. Buenos Aires: Kan Sasana Printer.

BARRETT, John C.

2001 «Agency, the Duality of Structure, and the Problem of the Archaeological Record», en Archaeological Theory Today, I. Hodder, ed., pp. 141-164. Cambridge: Polity Press.

BARTEL, Barth

1982 «A Historical Review of Technological and Archaeological Analysis of Mortuary Practices». Journal of Anthropological Archaeology 1: 32-58. doi: 10.1016/02784165(82)90007-1

BASCOPÉ CAERo, Víctor

2001 «El sentido de la muerte en la cosmovisión andina: el caso de los valles andinos de Cochabamba». Chungará 33 (2): 271-277. doi: 10.4067/S071773562001000200012

Bloch, Marc y Jonathan PARRY

1982 Death and the Regeneration of Life. Cambridge: Cambridge University Press.

Bordach, María de la Asunción

2006 «Interacciones étnicas e indicadores de desigualdad social en el Cementerio de La Falda (SJTIL 43), Tilcara, Jujuy». Estudios Atacameños 31: 115-128. doi: $10.4067 / \mathrm{S} 0718-10432006000100008$

Buikstra, Jane E. y Douglas K. Charles

1999 «Centering the Ancestors: Cemeteries, Mounds, and Sacred Landscapes of the Ancient North American Midcontinent», en Archaeologies of Landscape: Contemporary Perspectives, W. Ashmore y A. B. Knapp, eds., pp. 201-228. Oxford: Blackwell.

CANNON, Aubrey

1989 «The Historical Dimension in Mortuary Expression of Status and Sentiment». Current Anthropology 30 (4): 437-457.

CARR, Christopher

1995 «Mortuary Practices: Their Social, Philosophical-Religious, Circumstantial and Physical Determinants». Journal of Archaeological Method and Theory 2 (2): 105-200. doi: 10.1007/BF02228990

Casanova, Eduardo

1942 «El yacimiento arqueológico de Angosto Chico». Relaciones de la Sociedad Argentina de Antropología 3: 75-85.

Cigliano, Eduardo

1960 «Práctica funeraria en los diferentes entierros del yacimiento arqueológico de Huella (Prov. de Jujuy)». Revista del Instituto de Antropología 2-3: 263-272.

Coвo, Bernabé

1964 Historia del Nuevo Mundo [1653]. Madrid: Biblioteca de Autores Españoles. 


\section{Cremonte, María Beatriz}

2007 «El imperio perdura en las fronteras más lejanas. Instalaciones incaicas tardías en Jujuy, Argentina», en Sociedad y Cultura: las múltiples caras de sus fronteras, P. J. Chalé y L. A. Várguez Pasos, eds., pp. 331-344. México - Tempe: Universidad Autónoma de Yucatán - Arizona State University.

Cremonte, María Beatriz, Irma Lia Botto y R. ViÑa

2009 «Pigmentos y cuentas de collar hallados en contextos funerarios del sitio Esquina de Huajra (Quebrada de Humahuaca)», en Arqueometría Latinoamericana, O. M. Palacios et al. (eds.), pp. 183-189. Buenos Aires: Comisión Nacional de Energía Atómica.

Cremonte, María Beatriz, Sebastián M. Peralta y Alba Díaz

2008 «Petrografía de pastas cerámicas de Esquina de Huajra (Tumbaya, Jujuy). Las razones de su diversidad y consumo». Ponencia presentada en las IX Jornadas Regionales de Investigación en Humanidades y Ciencias Sociales. San Salvador de Jujuy.

Cremonte, María Beatriz, Sebastián M. Peralta y Agustina Scaro

2006-2007 «Esquina de Huajra (Tum 10, Dto. Tumbaya, Jujuy). Avances en el conocimiento de una instalación Humahuaca Inca y su integración en la historia prehispánica regional». Cuadernos del INAPL 21: 27-38.

Chaparro, María Gabriela

e.p. «Preferencias en el manejo cotidiano de rocas. Los artefactos líticos de los asentamientos estatales del sur de la Quebrada de Humahuaca y el valle Calchaquí medio (Argentina)», en Al Borde del Imperio. Paisajes Sociales en Areas Periféricas del Qollasuyu, V. Williams y M. B. Cremonte, eds.

Garay de Fumagalli, Mercedes y María Beatriz Cremonte

2007 «Contextos incaicos en momentos Hispano-Indígenas», en Actas XVI Congreso Nacional de Arqueología Argentina, tomo 1, pp. 507-512. San Salvador de Jujuy.

GHEGGi, María Soledad

2005 Análisis bioarqueológico y contextual en enterratorios arqueológicos. Un caso de estudio en Esquina de Huajra (Quebrada de Humahuaca - Jujuy, Argentina). Tesis de licenciatura inédita. Facultad de Filosofía y Letras, Universidad de Buenos Aires.

2005-2006 «Más allá de los huesos. El estudio integral de la evidencia de los entierros de Esquina de Huajra (Dto. Tumbaya, Quebrada de Humahuaca) en el contexto histórico regional». Arqueología 13: 47-78.

GidDENS, Anthony

1984 The Constitution of Society. Cambridge: Polity Press.

GLuCKMAn, Max

1937 «Mortuary Customs and the Belief in Survival After Death Among the SouthEastern Bantu». Bantu Studies 11: 117-136.

Goldstein, Lynn

1981 «One-Dimensional Archaeology and Multi-Dimensional People: Spatial Organization and Mortuary Analysis», en The Archaeology of Death, R. W. Chapman, I. Kinnes y K. Randsborg, eds., pp. 53-69. Cambridge: Cambridge University Press. 
HERTZ, Robert

1990 La muerte y la mano derecha [1907]. México: Patria.

LizÁRraga, Reginaldo de

2002 Descripción del Perú, Tucumán, Río de la Plata y Chile [1607]. Crónicas de América, 44. Madrid: Dastin.

Mendonça, Oslvaldo, María de la Asunción Bordach, M. Ester Albeck y Marta Ruiz

1997 «Collares de vidrio y ollas de barro. Comportamiento ante la Muerte en el Tilcara Hispano-Indígena inicial (Jujuy, Argentina)». Cuadernos 9: 175-202.

Mizoguchi, Koji

1993 «Time in the Reproduction of Mortuary Practices». World Archaeology 25 (2): 223-235.

Morris, Ian

1991 «The Archaeology of Ancestors: the Saxe/Goldstein Hypothesis Revisited». Cambridge Archaeological Journal 1 (2): 147-169.

Nielsen, Axel

2004 «Aproximación a la arqueología de la frontera tripartita Bolivia-Chile-Argentina». Chungará 36 (supl. especial): 861-878. doi: 10.4067/S0717-73562004000400026.

2006 «Plazas para los antepasados: descentralización y poder corporativo en las formaciones políticas preincaicas de los Andes circumpuneños». Estudios Atacameños 31: 63-89.

Nielsen, Axel y Lucio Boschi

2007 Celebrando con los Antepasados. Arqueología del Espacio Público en Los Amarillos. Quebrada de Humahuaca. Jujuy, Argentina. Jujuy: Mallku Ediciones.

OnOFRe Mamani, Luperio

2001 «Alma Imaña. Rituales mortuorios andinos en las zonas rurales Aymara de Puno Circunlacustre (Perú)». Chungará 33 (2): 233-244. doi: 10.4067/S071773562001000200007

Parker Pearson, Michael

1993 «The Powerful Dead: Archaeological Relationships Between the Living and the Dead». Cambridge Archaeological Journal 3: 203-229.

RAMSEY, Christopher

2007 Oxcal v. 4.0.2. Oxford Radiocarbon Accelerator Unit Research Lab for Archaeology. Oxford.

Salas, Alberto Mario

1945 «El antigal de Ciénaga Grande». Publicaciones de la Facultad de Filosofía y Letras de la Universidad de Buenos Aires. Museo Etnográfico Serie A ( $\mathrm{n}^{\circ}$ 5). Buenos Aires.

SÁNCHEZ, Sandra

1996 Fragmentos de un tiempo largo, Tilcara entre los siglos XV-XIX. Tesis de licenciatura inédita. Facultad de Humanidades y Ciencias Sociales, Universidad Nacional de Jujuy.

SELDEs, Verónica

2006 «Bioarqueología de las poblaciones prehistóricas de la quebrada de Humahuaca (Jujuy, Argentina)». Estudios Atacameños 31: 47-61. 
SicA, Gabriela

2006 Del Pukara al Pueblo de Indios. El proceso de construcción de la sociedad indígena colonial en Jujuy, Argentina. Siglo XVII. Tesis doctoral inédita. Universidad de Sevilla.

SiRACUSANO, Gabriela

2005 El poder de los colores. Buenos Aires: Fondo de Cultura Económica.

SuETTA, Juan Manuel.

1969 «Aportes a la arqueología de Volcán (Prov. de Jujuy)». Antiquitas 8: 1-6.

TARRAGÓ, Myriam

1984 «El Contrato Hispano-Indígena: La Provincia de Chicoana». Runa 14: 143-185.

TURNER, Victor

1995 The Ritual Process: Structure and Anti-Structure [1969]. Nueva York: Aldine de Gruyter.

VAN Kessel, Juan

2001 «El ritual mortuorio de los Aymara de Tarapacá como vivencia y crianza de la vida». Chungará 33 (2): 221-234. doi: 10.4067/S0717-73562001000200006 